\title{
Linear Programming Bounds on the Degree Distributions of LDPC Code Ensembles
}

\author{
Igal Sason \\ Department of Electrical Engineering \\ Technion, Haifa 32000, Israel \\ sason@ee.technion.ac.il
}

\begin{abstract}
This work considers the behavior of the degree distributions of capacity-approaching low-density parity-check (LDPC) code ensembles via linear programming (LP) bounds. These LP bounds are information-theoretic, and they apply to finite-length LDPC codes and to the asymptotic case of an infinite block length. Analytical solutions of these bounds are given in closed form, and the bounds are compared for the BEC with some specific degree distributions of capacity-achieving sequences of LDPC code ensembles. These LP bounds are shown to be informative and are easy to calculate. Due to space limitations, the derivation of the LP bounds is outlined, and the reader is referred to the full paper version [9] for complete proofs and further discussions.
\end{abstract}

Index Terms-Degree distributions, linear programming, lowdensity parity-check (LDPC) codes, memoryless binary-input output-symmetric (MBIOS) channels.

\section{INTRODUCTION}

Low-density parity-check (LDPC) codes form a class of powerful error-correcting codes which are efficiently encoded and decoded with low-complexity algorithms. In this paper, the following question is addressed:

Question: How do the degree distributions of capacityapproaching LDPC code ensembles behave as a function of the achievable gap (in rate) to capacity ?

Consider the case where the transmission takes place over a memoryless binary-input output-symmetric (MBIOS) channel. The behavior of the degree distributions of capacityapproaching LDPC code ensembles is addressed in the full paper version [9] via the derivation of some informationtheoretic bounds. The bounds are expressed in terms of the gap (in rate) to capacity with a target bit (or block) error probability. These linear programming (LP) upper bounds on the degree distributions of LDPC code ensembles are general with respect to the decoding algorithm, and they also hold for ensembles of finite-length codes or for the asymptotic case of an infinite block length.

In this context, two LP problems are formulated in [1] for optimizing the degree distributions of finite-length LDPC code ensembles whose transmission takes place over a BEC, and also a convex optimization problem is formulated in [2] for optimizing the degree distributions of LDPC code ensembles with the goal of obtaining a good tradeoff between performance and decoding complexity. It is noted that the LP-based optimizations in [1] and [2] hold under belief propagation (BP) decoding, whereas the LP bounds which are derived in this paper are information-theoretic bounds which hold under ML decoding or any sub-optimal decoding algorithm. Although the degree distributions of the parity-check nodes are often set to be regular (or almost regular), and the irregularity often refers to the degree distributions of the variable nodes, this is not necessarily the case for capacity-approaching ensembles. For example, [7, Section VI] introduces some capacity-achieving sequences of accumulate-repeat-accumulate code ensembles for the BEC, which also possess a bounded complexity per information bit under BP decoding; they are designed in a way where the degree distributions of the LDPC code ensembles after a proper graph reduction (as explained in [7, Section II]) are self-matched and are both irregular. The irregularity of the parity-check degree distributions in the design of LDPC codes appears to be useful in various cases under BP decoding (see, e.g., [1], [3], [6], and [10]).

\section{LP BOUNDS ON THE DEGREE DISTRIBUTIONS OF LDPC CODE ENSEMBLES}

This section provides LP bounds on the degree distributions of LDPC code ensembles. Due to space limitations, the proofs are outlined here, and the reader is referred to [9] for full proofs and further discussions. These bounds are formulated in terms of the target bit error probability and the gap (in rate) to capacity required to achieve this target. The following LP bounds refer to the node and edge perspectives of the degree distributions, and they provide upper bounds on the fraction of edges or nodes up to a degree $k$ where $k$ is a positive integer. The LP bounds that are introduced in this section hold under ML decoding, and they are therefore general in terms of the decoding algorithm. These LP bounds apply to finite-length LDPC code ensembles, and also to the asymptotic case of an infinite block length. Analytical solutions of these LP bounds are provided in closed form, and these bounds are also compared with some capacity-achieving sequences of LDPC code ensembles for the BEC under BP decoding. In the continuation to this section, we denote

$$
\begin{aligned}
g_{1} & \triangleq \mathbb{E}\left[\tanh ^{2}(L / 2)\right] \\
& =\int_{0}^{\infty} a(l)\left(1+e^{-l}\right) \tanh ^{2}\left(\frac{l}{2}\right) d l
\end{aligned}
$$

where $L$ is a random variable which refers to the log-likelihood ratio (LLR) at the channel output, given that the binary input symbol to the channel is zero, the symbol $\mathbb{E}$ denotes the 
statistical expectation with respect to $L$, and $a$ designates the symmetric probability density function $(p d f)$ of $L$ (i.e., for an MBIOS channel, the equality $a(l)=e^{l} a(-l)$ holds for $l \in \mathbb{R}$ [8, Chapter 3]). Hence, $g_{1}$ in (1) is a parameter which only depends on the MBIOS communication channel (but not on the code ensemble).

In the following, we consider ensembles of binary LDPC codes. Following standard notation, let $\lambda_{i}$ and $\rho_{i}$ denote the fraction of edges attached, respectively, to variable and paritycheck nodes of degree $i$. Let $\Lambda_{i}$ and $\Gamma_{i}$ denote, respectively, the fraction of variable and parity-check nodes of degree $i$. The LDPC code ensemble is characterized by a triple $(n, \lambda, \rho)$, where $n$ designates the block length of the codes, and $\lambda(x) \triangleq$ $\sum_{i} \lambda_{i} x^{i-1}$ and $\rho(x) \triangleq \sum_{i} \rho_{i} x^{i-1}$ represent, respectively, the left and right degree distributions from the edge perspective. Equivalently, this ensemble is also characterized by the triple $(n, \Lambda, \Gamma)$ where $\Lambda(x) \triangleq \sum_{i} \Lambda_{i} x^{i}$ and $\Gamma(x) \triangleq \sum_{i} \Gamma_{i} x^{i}$ represent, respectively, the left and right degree distributions from the node perspective.

The following LP bounds are separated into four categories:

- LP1: 'LP1' forms an LP upper bound on the degree distribution of the parity-check nodes for LDPC code ensembles whose transmission takes place over an MBIOS channel, and let $C$ designate the channel capacity in bits per channel use. The first version of this bound gives an upper bound on the fraction of parity-check nodes up to an arbitrary degree $k$ (where $k \geq 1$ is an integer) as a function of the achievable rate (and its fraction from the channel capacity) with a given bit error probability $P_{\mathrm{b}}$. From [9], the following optimization problem follows:

$$
\begin{aligned}
& \text { maximize } \sum_{i=1}^{k} \Gamma_{i}, \quad k=1,2, \ldots \\
& \text { subject to } \\
& \left\{\begin{array}{l}
\sum_{i=1}^{\infty}\left\{\left[1-h_{2}\left(\frac{1-g_{1}^{\frac{i}{2}}}{2}\right)\right] \Gamma_{i}\right\} \leq \frac{\varepsilon C+h_{2}\left(P_{\mathrm{b}}\right)}{1-(1-\varepsilon) C} \\
\sum_{i=1}^{\infty} \Gamma_{i}=1 \\
\Gamma_{i} \geq 0, \quad i=1,2, \ldots
\end{array}\right.
\end{aligned}
$$

where the optimization variables are $\left\{\Gamma_{i}\right\}_{i \geq 1}$, and $k$ is a parameter. The function $h_{2}$, which appears in the first inequality constraint of the LP1 bound, designates the binary entropy function, i.e.,

$h_{2}(x)=-x \log _{2}(x)-(1-x) \log _{2}(1-x), \quad 0 \leq x \leq 1$

and $g_{1}$ is introduced in (1).

Outline of the proof:

Let us assume in the following that the transmission of a binary linear block code takes place over an MBIOS channel. Let $L$ designate the LLR at the channel output, given that the channel input is zero, and let $a$ designate the probability density function $(p d f)$ of $L$.

Let us consider a binary linear block code $\mathcal{C}$ of length $n$ and rate $R$, and let $\mathbf{X}$ and $\mathbf{Y}$ be the transmitted codeword and received sequence, respectively. Assume that the codewords of $\mathcal{C}$ have no bits which are set a-priori to zero. We first assume that the code $\mathcal{C}$ is represented by a parity-check matrix $H$ which is full rank (i.e., the rows of $H$ are linearly independent). For an arbitrary full-rank parity-check matrix of a binary linear block code $\mathcal{C}$, let $\Gamma_{k}$ designate the fraction of the parity-checks involving $k$ variables, and let $\Gamma(x) \triangleq \sum_{k} \Gamma_{k} x^{k}$. The following lower bound on the conditional entropy of the transmitted codeword, given the received sequence at the channel output, holds (see [11]):

$$
\frac{H(\mathbf{X} \mid \mathbf{Y})}{n} \geq R-C+\frac{1-R}{2 \ln 2} \sum_{p=1}^{\infty} \frac{\Gamma\left(g_{p}\right)}{p(2 p-1)}
$$

where

$$
g_{p} \triangleq \int_{0}^{\infty} a(l)\left(1+e^{-l}\right) \tanh ^{2 p}\left(\frac{l}{2}\right) d l, \quad p \in \mathbb{N} .
$$

The above lower bound on the conditional entropy in (2) holds for any representation of the code by a full-rank parity-check matrix. Note that the derivation of the lower bound in (2) relies on the assumption that the paritycheck matrix is full rank. Though it seems like a feasible requirement for specific binary linear block codes, this poses a problem when considering ensembles of LDPC codes. In the latter case, a parity-check matrix which corresponds to a randomly chosen bipartite graph with a given pair of degree distributions may not be full rank. To this end, the following lemma is provided in [9]:

Lemma 1: For (regular/ irregular) ensembles of binary LDPC codes, the inequality in (2) stays valid for every code from the ensemble with the following modifications:

- The rate $R$ of the code is replaced with the design rate $\left(R_{\mathrm{d}}\right)$ of the ensemble.

- The sequence $\left\{\Gamma_{k}\right\}$ refers to the degree distribution of the parity-check nodes of the ensemble (where the representation of a code by a parity-check matrix, with the given degree distribution, possibly includes some linearly dependent rows).

Proof: See [9, Appendix I].

The derivation of the first inequality constraint in the LP1 problem relies on the extension of the validity of (2) to the case where the parity-check matrix is not necessarily full rank, as given in Lemma 1, and it also relies on the inequality $g_{p} \geq\left(g_{1}\right)^{p}$ which holds for every $p \geq 1$ (this inequality follows directly from Jensen's inequality). Finally, its proof is completed by relying on the power series expansion of the binary entropy function

$$
h_{2}(x)=1-\frac{1}{2 \ln 2} \sum_{p=1}^{\infty} \frac{(1-2 x)^{2 p}}{p(2 p-1)}, \quad 0 \leq x \leq 1
$$

and the Fano inequality, from which it follows that

$$
\frac{H(\mathbf{X} \mid \mathbf{Y})}{n} \leq h_{2}\left(P_{\mathrm{b}}\right)
$$

For a detailed proof of the LP1 bound, the reader is referred to the derivation of [9, Eq. (61)].

From the standard transition from the degree distributions from the node perspective to degree distributions from the 
edge perspective (see [8]), the following equality holds:

$$
\Gamma_{i}=\frac{\rho_{i}}{i}\left(\sum_{j=1}^{\infty} \frac{\rho_{j}}{j}\right)^{-1} .
$$

The substitution of this equality in the first constraint of the above LP bound gives the following optimization problem for the degree distribution of the parity-check nodes from the edge perspective (i.e., we get an upper bound on the fraction of edges which are connected to parity-check nodes up to degree $k \geq 1$ ):

$$
\begin{aligned}
& \text { maximize } \sum_{i=1}^{k} \rho_{i}, \quad k=1,2, \ldots \\
& \text { subject to } \\
& \left\{\begin{array}{l}
\sum_{i=1}^{\infty}\left\{\left[1-h_{2}\left(\frac{1-g_{1}^{\frac{i}{2}}}{2}\right)\right] \frac{\rho_{i}}{i}\right\} \leq \frac{\varepsilon C+h_{2}\left(P_{\mathrm{b}}\right)}{1-(1-\varepsilon) C} \sum_{i=1}^{\infty} \frac{\rho_{i}}{i} \\
\sum_{i=1}^{\infty} \rho_{i}=1 \\
\rho_{i} \geq 0, \quad i=1,2, \ldots
\end{array}\right.
\end{aligned}
$$

where the optimization variables are $\left\{\rho_{i}\right\}_{i \geq 1}$. These two LP bounds are valid under ML decoding (hence, they also hold under any other decoding algorithm). These bounds hold for finite-length codes and also for the asymptotic case of an infinite block length. Based on strong duality for LP problems (which holds unless the primal and the dual LP problems are both not feasible [4]), an analytical solution of the LP1 bound is given in [9, Appendix VII]. This bound is also tightened in [9, Appendix VII] for the BEC, followed by its analytical solution.

- LP2: 'LP2' provides a universal LP upper bound on the degree distribution of the parity-check nodes for LDPC code ensembles as a function of the required achievable rate (and its fraction $\varepsilon$ from the channel capacity) with a required bit error probability $P_{\mathrm{b}}$. This bound gets the form:

$$
\begin{aligned}
& \text { maximize } \sum_{i=1}^{k} \rho_{i}, \quad k=1,2, \ldots \\
& \text { subject to } \\
& \left\{\begin{array}{l}
\sum_{i=1}^{\infty}\left\{\left[1-h_{2}\left(\frac{1-C^{\frac{i}{2}}}{2}\right)\right] \frac{\rho_{i}}{i}\right\} \leq \frac{\varepsilon C+h_{2}\left(P_{\mathrm{b}}\right)}{1-(1-\varepsilon) C} \sum_{i=1}^{\infty} \frac{\rho_{i}}{i} \\
\sum_{i=1}^{\infty} \rho_{i}=1 \\
\rho_{i} \geq 0, \quad i=1,2, \ldots
\end{array}\right.
\end{aligned}
$$

where the optimization variables are $\left\{\rho_{i}\right\}_{i \geq 1}$, and the bound holds under the same conditions as in the previous item. However, the LP2 bound is universal in the sense that it holds for all MBIOS channels which exhibit a given capacity $C$. The LP2 bound follows from the same reasoning which leads to the LP1 bound with the additional property which states that $g_{1} \geq C$ for all MBIOS channels with capacity $C$, and this inequality holds with equality for a BEC. The LP2 bounds from the node and edge perspectives are similar to the corresponding formulations of the LP1 bound, except of the replacement of $g_{1}$ in the LP1 bounds with the channel capacity $C$. Hence, their analytical solution is similar to the LP1 bound, except of this simple replacement.

- LP3: 'LP3' provides an LP upper bound on the degree distribution of the variable nodes (from the edge perspective) for LDPC code ensembles whose transmission takes place over an MBIOS channel. This bound provides an upper bound on the fraction of edges which are connected to variable nodes up to degree $k$ for a parameter $k \geq 2$, and it is expressed in terms of the required achievable rate (and its gap to capacity) with a given bit error probability $P_{\mathrm{b}}$. This LP bound gets the following form (see [9]):

$$
\begin{aligned}
& \text { maximize } \sum_{i=2}^{k} \lambda_{i}, \quad k=2,3, \ldots \\
& \text { subject to } \\
& \left\{\begin{array}{l}
\sum_{i=2}^{\infty} \frac{\lambda_{i}}{i} \leq \frac{\ln \left(\frac{1}{g_{1}}\right)}{2(1-C)\left(1+\frac{\varepsilon C}{1-C}\right) \ln \left(\frac{1}{1-2 h_{2}^{-1}\left(\frac{1-C-h_{2}\left(P_{\mathrm{b}}\right)}{1-(1-\varepsilon) C}\right)}\right)} \\
\sum_{i=2}^{\infty} \lambda_{i}=1 \\
\lambda_{i} \geq 0, \quad i=2,3, \ldots
\end{array}\right.
\end{aligned}
$$

where the optimization variables are $\left\{\lambda_{i}\right\}_{i \geq 2}$. The bound is valid under ML decoding (or any other decoding algorithm). It holds for finite block-length as well as in the asymptotic case where we let the block length tend to infinity. The first inequality constraint in the LP3 bound follows from the improved lower bound on the average degree of the variable nodes, as given in [9, Theorem 1], and the other two constraints are trivial.

- LP4: 'LP4' provides a universal LP upper bound on the degree distribution of the variable nodes for LDPC code ensembles (from the edge perspective). It gets the following form (see [9]):

maximize $\sum_{i=2}^{k} \lambda_{i}, \quad k=2,3, \ldots$
subject to
$\left\{\begin{array}{l}\sum_{i=2}^{\infty} \frac{\lambda_{i}}{i} \leq \frac{\ln \left(\frac{1}{C}\right)}{2(1-C)\left(1+\frac{\varepsilon C}{1-C}\right) \ln \left(\frac{1}{1-2 h_{2}^{-1}\left(\frac{1-C-h_{2}\left(P_{\mathrm{b}}\right)}{1-(1-\varepsilon) C}\right)}\right)} \\ \sum_{i=2}^{\infty} \lambda_{i}=1 \\ \lambda_{i} \geq 0, \quad i=2,3, \ldots\end{array}\right.$

where the optimization variables are $\left\{\lambda_{i}\right\}_{i \geq 2}$. This bound holds for all MBIOS channels with a given capacity $C$. The only difference between the LP3 and LP4 bounds is that the parameter $g_{1}$ in the first inequality constraint of the LP3 bound is replaced with the capacity $C$ in the LP4 bound (similarly to the difference between the LP1 and LP2 bounds). 
Analytical solutions for the LP1 and LP2 bounds: The LP1 problem can be expressed in the following equivalent form:

$$
\begin{aligned}
& \text { maximize } \sum_{i=1}^{k} \rho_{i}, \quad k=1,2, \ldots \\
& \text { subject to } \\
& \left\{\begin{array}{l}
\sum_{i=1}^{\infty} d_{i} \rho_{i} \leq 0 \\
d_{i} \triangleq \frac{1}{i}\left[1-h_{2}\left(\frac{1-g_{1}^{\frac{i}{2}}}{2}\right)-\frac{\varepsilon C+h_{2}\left(P_{\mathrm{b}}\right)}{1-(1-\varepsilon) C}\right] \\
\sum_{i=1}^{\infty} \rho_{i}=1 \\
\rho_{i} \geq 0, \quad i=1,2, \ldots
\end{array}\right.
\end{aligned}
$$

An analytical solution for the LP1 bound is obtained in [9, Appendix VII] (via the use of strong Lagrange duality).

In the following, the final solution of the LP1 bound is presented. To this end, note that for indices $i$ large enough, $d_{i}<0$ and also $\lim _{i \rightarrow \infty} d_{i}=0$. Let $d^{*} \triangleq \min _{i>1} d_{i}$ be the minimal value of this sequence, and let $i=l$ be the corresponding index of $d_{i}$ which achieves this minimal value of the sequence $\left\{d_{i}\right\}$. Clearly, $d^{*}<0$. The resulting closedform solution for the LP1 bound gets the following form:

- For integer values of $k$ which satisfy $k \leq k_{0}$ where

$$
k_{0} \triangleq \frac{2 \ln \left(\frac{1}{1-2 h_{2}^{-1}\left(\frac{1-C-\delta}{1-(1-\varepsilon) C}\right)}\right)}{\ln \left(\frac{1}{g_{1}}\right)} .
$$

The LP1 upper bound is equal to $-\frac{d^{*}}{d_{k}-d^{*}}$.

- For values of $k$ larger than or equal to $k_{0}$, the LP1 upper bound is equal to 1 (hence, it becomes trivial).

A similar solution is obtained for the LP2 bound where the only difference is that $g_{1}$ in the definition of the sequence $\left\{d_{i}\right\}$ is replaced by the channel capacity $C$. These analytical solutions match the numerical solutions obtained via [5].

Example 1: [A comparison of the LP1 bound and capacity-achieving LDPC code ensembles over the BEC] In the following, we compare the LP1 bound for the BEC and the degree distributions of two capacity-achieving sequences of LDPC code ensembles under iterative message-passing decoding.

The first capacity-achieving sequence for the BEC refers to the heavy-tail Poisson distribution, and it was introduced in [6, Section IV], [10] (see also [8, Problem 3.20]). The second capacity-achieving sequence refers to the right-regular LDPC code ensembles [10], based also on the analysis in the proof of [9, Proposition 1].

This first capacity-achieving sequence is obtained via the pair of degree distributions

$$
\begin{aligned}
& \hat{\lambda}_{\alpha}(x)=-\frac{1}{\alpha} \cdot \ln (1-x)=\frac{1}{\alpha} \sum_{i=1}^{\infty} \frac{x^{i}}{i} \\
& \rho_{\alpha}(x)=e^{\alpha(x-1)}=e^{-\alpha} \sum_{i=0}^{\infty} \frac{\alpha^{i} x^{i}}{i !}
\end{aligned}
$$

which satisfies the equality $\hat{\lambda}_{\alpha}\left(1-\rho_{\alpha}(1-x)\right)=x$ for all $\alpha>0$. Starting with the heavy-tail Poisson distribution as above and proceeding along the lines in [8, Section 3.15], the following two steps are performed for the construction of capacity-approaching LDPC code ensembles for the BEC:

- The degree distribution $\hat{\lambda}_{\alpha}(x)$ is truncated so that it consists of the first $N$ terms of its Taylor series expansion (up to and including the term $x^{N-1}$ ).

- The truncated power series $\hat{\lambda}_{\alpha}^{(N)}(x)$ is normalized so that it is equal to 1 at $x=1$. The left degree distribution (from the edge perspective) is then equal to $\lambda_{\alpha}^{(N)}(x)=\frac{\hat{\lambda}_{\alpha}^{(N)}(x)}{\hat{\lambda}_{\alpha}^{(N)}(1)}$. The right degree distribution, $\rho_{\alpha}(x)$, is not modified.

This procedure provides the following degree distributions:

$$
\begin{aligned}
\lambda_{i} & =\frac{1}{H(N-1)(i-1)}, \quad i=2,3, \ldots N \\
\rho_{i} & =\frac{e^{-\alpha} \alpha^{i-1}}{(i-1) !}, \quad i=1,2, \ldots
\end{aligned}
$$

where $H(k) \triangleq \sum_{i=1}^{k} \frac{1}{i}$ for $k \geq 1$ is a truncated harmonic sum. Straightforward calculus shows that the design rate of the corresponding LDPC code ensemble is equal to

$$
R_{\mathrm{d}}(\alpha, N)=1-\frac{N H(N-1)\left(1-e^{-\alpha}\right)}{(N-1) \alpha} .
$$

We need to determine the parameters $\alpha$ and $N$ so that the design rate in (5) forms (at least) a fraction $1-\varepsilon$ of the capacity of the BEC. Let $p$ designate the erasure probability of the channel, and let $r=(1-\varepsilon)(1-p)$ be the lower bound on the required design rate. We need to choose $\alpha$ and $N$ to satisfy the inequality $R_{\mathrm{d}}(\alpha, N) \geq r$ with vanishing bit erasure probability under BP decoding. Similarly to the calculations in [8, Example 3.88], the satisfiability of the inequality

$$
\frac{\hat{\lambda}_{\alpha}^{(N)}(1)}{1-\hat{\lambda}_{\alpha}^{(N)}(1)}\left(\frac{\int_{0}^{1} \rho_{\alpha}(x) \mathrm{d} x}{\int_{0}^{1} \hat{\lambda}_{\alpha}^{(N)}(x) \mathrm{d} x}-1\right) \leq \varepsilon
$$

implies this requirement, and straightforward algebra gives (see [9, Example 6])

$$
\alpha=\frac{H(N-1)}{1-r}, \quad N=\left\lceil\frac{1-r}{\varepsilon r}\right\rceil+1 .
$$

In the following, we calculate the heavy-tail Poisson distribution in (4) with the choice of parameters in (6). The resulting degree distribution of the parity-check nodes (from the edge perspective) is compared with the LP1 bound for the BEC where the analytical solution of this bound is given in $[9$, Appendix VII].

Comparisons between the heavy-tail Poisson distribution and the LP1 bound are shown in Figure 1. We note that the LP1 bound is an upper bound on the parity-check degree distribution which is valid under ML decoding (and hence, it is general for any decoding algorithm), whereas the heavytail Poisson distribution is designed to achieve a certain gap to capacity under BP decoding. We also show in this figure the fixed degree of the parity-check nodes for the rightregular LDPC code ensemble; this calculation is done via [9, Eqs. (112), (113) and (118)] where the right degree is equal to $a_{\mathrm{R}}=\left\lceil\frac{1}{\alpha}\right\rceil+1$. Although the latter case corresponds to a step 

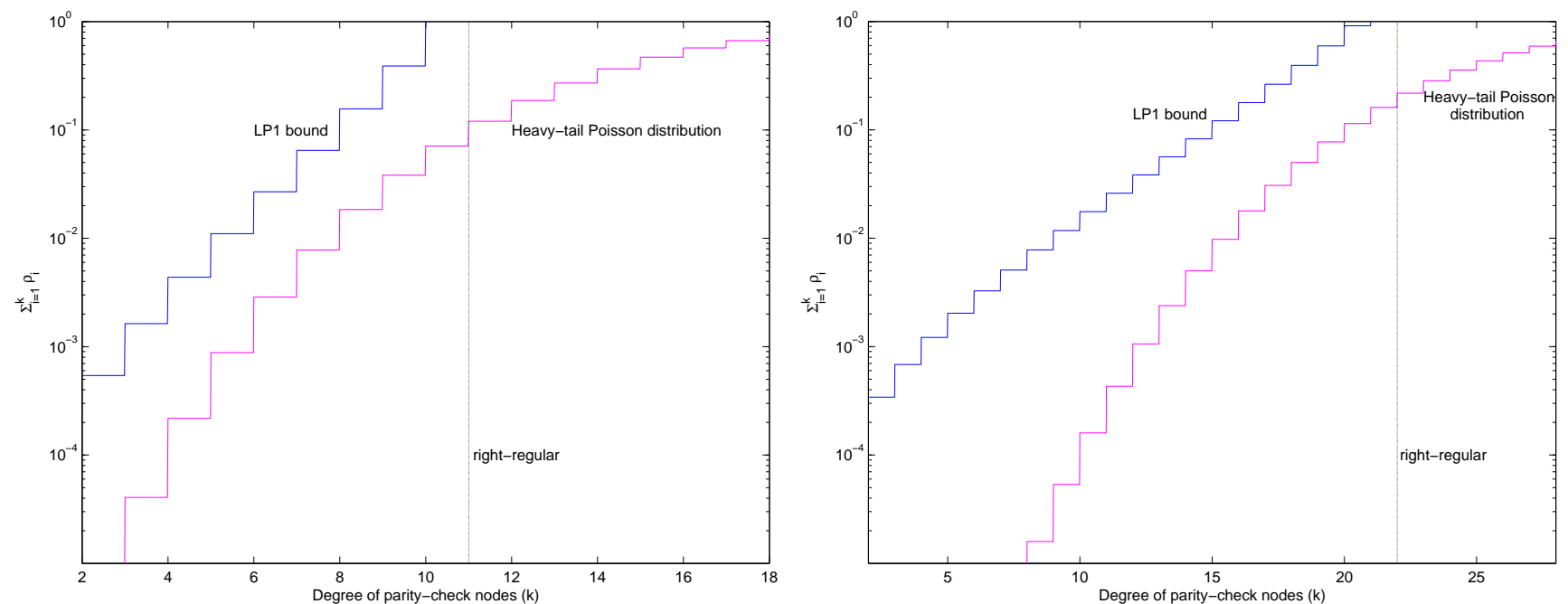

Fig. 1. A comparison between the LP1 bound and the heavy-tail Poisson degree distribution in (4) and (6), and the parity-check degree distribution of the right-regular LDPC ensemble (it is calculated via [9, Eqs. (112), (113) and (118)] where the right degree is equal to $a_{\mathrm{R}}=[1 / \alpha\rceil+1$ ). This comparison refers to a BEC whose capacity is one-half (upper plot) and three-quarters (lower plot) bits per channel use, and the setting where $99.9 \%$ of the channel capacity is achieved under BP decoding with vanishing bit erasure probability. The stair functions correspond to the fraction of edges which are attached to parity-check nodes whose degrees are at most $k$ for a positive integer $k$.

function, the degree where this function is switched from zero to one provides an indication to the reasonable tightness of the LP1 upper bound with respect to the value of the parity-check degree $k$ where this upper bound is close to 1 .

Analytical solutions for the LP3 and LP4 bounds: As shown in [9, Section V.C], the closed-form solution of the LP3 bound is given by

$\min \left\{1, \frac{k \ln \left(\frac{1}{g_{1}}\right)}{2(1-C)\left(1+\frac{\varepsilon C}{1-C}\right) \ln \left(\frac{1}{1-2 h_{2}^{-1}\left(\frac{1-C-h_{2}\left(P_{b}\right)}{1-(1-\varepsilon) C}\right)}\right)}\right\}$.

The closed form solution of the LP4 bound is the same as in (7), except of replacing the parameter $g_{1}$ by $C$.

Example 2 (LP3 bound): Consider LDPC code ensembles whose design rate is one-half bit per channel use, and whose transmission takes place over a BIAWGN channel. Lets assume that we wish to find upper bounds on the fraction of edges up to degree $k$ (for a parameter $k \geq 2$ ) for the setting of a bit error probability of (at most) $P_{\mathrm{b}}=10^{-10}$ under ML decoding (or any sub-optimal decoding algorithm) at $\frac{E_{\mathrm{b}}}{N_{0}}=0.188 \mathrm{~dB}$. This implies a gap to capacity which is equal to $\varepsilon=1.42 \times 10^{-4}$. From (7), we obtain the following inequalities (which are also verified numerically via [5]):

$$
\begin{aligned}
& \lambda_{2} \leq 0.2683 \\
& \lambda_{2}+\lambda_{3} \leq 0.4025 \\
& \lambda_{2}+\lambda_{3}+\lambda_{4} \leq 0.5367 \\
& \lambda_{2}+\lambda_{3}+\lambda_{4}+\lambda_{5} \leq 0.6709 \\
& \lambda_{2}+\lambda_{3}+\lambda_{4}+\lambda_{5}+\lambda_{6} \leq 0.8051 \\
& \lambda_{2}+\lambda_{3}+\lambda_{4}+\lambda_{5}+\lambda_{6}+\lambda_{7} \leq 0.9392 \\
& \lambda_{2}+\lambda_{3}+\lambda_{4}+\lambda_{5}+\lambda_{6}+\lambda_{7}+\lambda_{8} \leq 1.0000 .
\end{aligned}
$$

A comparison of these numerical results with those presented in Fig. 1 for the same value of $\frac{E_{\mathrm{b}}}{N_{0}}$ shows a big difference between the two upper bounds on the sequences $\left\{\lambda_{i}\right\}$ and $\left\{\rho_{i}\right\}$. This difference is well expected in light of the bounds in $[9$, Corollary 2] where for every finite degree $i$, the upper bounds on $\lambda_{i}$ and $\rho_{i}$ scale like $\frac{1}{\log \frac{1}{\varepsilon}}$ and $\frac{\varepsilon}{\log \frac{1}{\varepsilon}}$, respectively. We note that this difference is not an artifact of the bounding technique, as is demonstrated in [9, Proposition 1] for the BEC.

\section{REFERENCES}

[1] A. Amraoui, A. Montanari and R. Urbanke, "How to find good finitelength codes: from art towards science," European Transactions on Telecommunications, vol. 18, no. 5, pp. 491-508, April 2007.

[2] M. Ardakani, B. Smith, W. Yu and F. R. Kschischang, "Complexityoptimized low-density parity-check codes," Proceedings of the FortyThird Annual Allerton Conference on Communication, Control and Computing, pp. 45-54, Urbana-Champaign, Illinois, USA, September 2005.

[3] M. H. Azmi, J. Yuan, J. Ning and H. Q. Huynh, "Improved bilayer LDPC codes using irregular check node degree distribution," Proceedings 2008 IEEE International Symposium on Information Theory, pp. 141-145, Toronto, Canada, July 2008.

[4] S. Boyd and L. Vanderberghe, Convex Optimization, Cambridge Press, 2004. [Online]. Available: http://www.stanford.edu/ boyd/cvxbook/.

[5] M. Grant and S. Boyd. CVX: Matlab software for disciplined convex programming, August 2008. [Online]. Available: http://stanford.edu/ $\sim$ boyd/cvx.

[6] M. G. Luby, M. Mitzenmacher, M. A. Shokrollahi and D. A. Spielman, "Efficient erasure-correcting codes," IEEE Trans. on Information Theory, vol. 47, no. 2, pp. 569-584, February 2001.

[7] H. D. Pfister and I. Sason, "Accumulate-repeat-accumulate codes: Capacity-achieving ensembles of systematic codes for the erasure channel with bounded complexity," IEEE Trans. on Information Theory, vol. 53, no. 6, pp. 2088-2115, June 2007.

[8] T. Richardson and R. Urbanke, Modern Coding Theory, Cambridge University Press, 2008. [Online]. Available: http://lthcwww.epfl.ch/mct/ index.php.

[9] I. Sason, "On universal properties of capacity-approaching LDPC code ensembles," IEEE Trans. on Information Theory, vol. 55, no. 7, July 2009.

[10] A. Shokrollahi, "Capacity-achieving sequences," IMA Volume in Mathematics and its Applications, vol. 123, pp. 153-166, 2000.

[11] G. Wiechman and I. Sason, "Parity-check density versus performance of binary linear block codes: New bounds and applications," IEEE Trans. on Information Theory, vol. 53, no. 2, pp. 550-579, February 2007. 